\title{
Soft-x-ray spectroscopic investigation of ferromagnetic Co-doped ZnO
}

\author{
S. Krishnamurthy, C. McGuinness, ${ }^{\text {a) }}$ L. S. Dorneles, M. Venkatesan, J. M. D. Coey, \\ J. G. Lunney, and C. H. Patterson ${ }^{b}$ \\ School of Physics, Trinity College Dublin, Dublin 2, Ireland \\ K. E. Smith, T. Learmonth, and P. -A. Glans \\ Department of Physics, Boston University, 590 Commonwealth Avenue, Massachusetts 02215 \\ T. Schmitt ${ }^{\mathrm{c})}$ \\ Laboratory of Materials and Semiconductor Physics, Royal Institute of Technology, Electrum 229, \\ S-164 40 Kista, Sweden \\ and MAX-laboratory, Lund University, Box 118, S-22100 Lund, Sweden \\ J. -H. Guo \\ Advanced Light Source, Lawrence Berkeley National Laboratory, Berkeley, California 94720
}

(Presented on 2 November 2005; published online 21 April 2006)

\begin{abstract}
The electronic properties of cobalt-doped $\mathrm{ZnO}$ were investigated through site-selective and element-sensitive x-ray-absorption spectroscopy in the vicinity of the Co $L_{2,3}$ edge, the oxygen $K$ edge, and at the $\mathrm{Zn} L_{3}$ edge. The spectroscopic measurements of the ferromagnetic cobalt-doped $\mathrm{ZnO}$ films appear to have additional components in the $\mathrm{O} K$ edge $\mathrm{x}$-ray-absorption spectrum not observed in the undoped films. The observed features may derive from both hybridization with unoccupied Co $3 d$ states and also from lattice defects such as oxygen vacancies. Only minor changes in the $\mathrm{Zn} L_{3}$ edge spectra were observed. These observations are consistent with a polaron percolation model in which the ferromagnetic coupling is mediated by shallow donor electrons trapped in oxygen vacancies and couples the Co atoms substituted on $\mathrm{Zn}$ sites in the hexagonal wurtzite ZnO structure. () 2006 American Institute of Physics. [DOI: 10.1063/1.2165916]
\end{abstract}

Dilute magnetic semiconductors (DMS) are an especially interesting area of research because of their potential applications in spin electronics and magneto-optics. DMS based on the wide-band-gap semiconductor $\mathrm{ZnO}$ doped with a transition metal have been predicted theoretically to be good candidates for room-temperature ferromagnetism. ${ }^{1}$ Subsequently Ueda et $a l^{2}$ reported ferromagnetism in Codoped $\mathrm{ZnO}$. Following their work many conflicting reports have attributed the origin of ferromagnetic behavior as being due to substitution by $\mathrm{Co}$ atoms in the $\mathrm{ZnO}^{2-6}$ or due to clustering of $\mathrm{Co}$ atoms in secondary phases that are ferromagnetic; ${ }^{7}$ while others report no ferromagnetic behavior even though Co occupies the substitutional sites. ${ }^{8}$ Three different mechanisms have been proposed to explain the origin of ferromagnetism in transition-metal-doped $\mathrm{ZnO}$ : (I) A model of ferromagnetism where there is an exchange interaction mediated by carriers in the valence or conduction band and the localized moment of the ion, ${ }^{1}$ (II) a double exchange mechanism in which hopping of $3 d$ electrons from one ion to the next results in ferromagnetic behavior. ${ }^{9}$, and (III) an impurity band model ${ }^{10}$ where localized ionic moments create magnetic polarons in a defect-related donor impurity band. Clearly, it is desirable to find a way to distinguish between these models and to decide whether any of them is the correct model for doped $\mathrm{ZnO}$ systems. Hence, it

\footnotetext{
${ }^{a)}$ Electronic mail: cormac.mcguinness@tcd.ie

${ }^{b)}$ Present address: Quantum Theory Project, University of Florida, P.O. Box 118435, FI 32611-8435, USA.

${ }^{c}$ Present address: Swiss Light Source, Paul Scherrer Institut, CH-5232 Villigen PSI, Switzerland.
}

is important to verify that the transition-metal dopants occupy the $\mathrm{Zn}$ site and that ferromagnetism can result. In light of earlier reports such as that of Wi et al. ${ }^{8}$ substitutional cobalt may be a necessary but not sufficient condition for ferromagnetism. This realization may reconcile some of the apparently conflicting experimental reports.

In this report, element specific soft-x-ray-absorption techniques were used to help address the origin of roomtemperature ferromagnetism in Co-doped $\mathrm{ZnO}$ grown by pulsed laser deposition (PLD). The power of x-rayabsorption spectroscopy (XAS) at the Co $L_{2,3}$ edge allows the identification of the oxidation state and site symmetry of the cobalt ions. The $\mathrm{O} K$ edge and $\mathrm{Zn} L$ edge XAS spectra were also measured and it is believed that oxygen defectrelated features in the $\mathrm{O} K$ edge absorption are observed. All samples studied were thin films prepared by PLD. Targets were first prepared by thoroughly mixing the stoichiometric high-purity oxide powders; these were then pressed into pellets and sintered at $1100{ }^{\circ} \mathrm{C}$ for $24 \mathrm{~h}$. Films were deposited on (0001) $\mathrm{Al}_{2} \mathrm{O}_{3}$ substrates maintained at $450{ }^{\circ} \mathrm{C}$ during deposition in an oxygen atmosphere of $10^{-5}$ Torr. A KrF excimer laser operating at $248 \mathrm{~nm}, 10 \mathrm{~Hz}$ repetition rate, and a fluence of $1.8 \mathrm{~J} \mathrm{~cm}^{-2}$ was used to ablate the pressed powder. Details of sample growth were previously reported by Venkatesan et al. ${ }^{5}$ It should be noted that the observed ferromagnetic behavior is very sensitive to the growth conditions. X-ray-diffraction measurements confirm the $c$-axis-oriented growth of wurtzite zinc oxide $(w-\mathrm{ZnO})$ free from secondary phases. All the films are approximately $250 \mathrm{~nm}$ thick as determined by using optical reflectivity at 


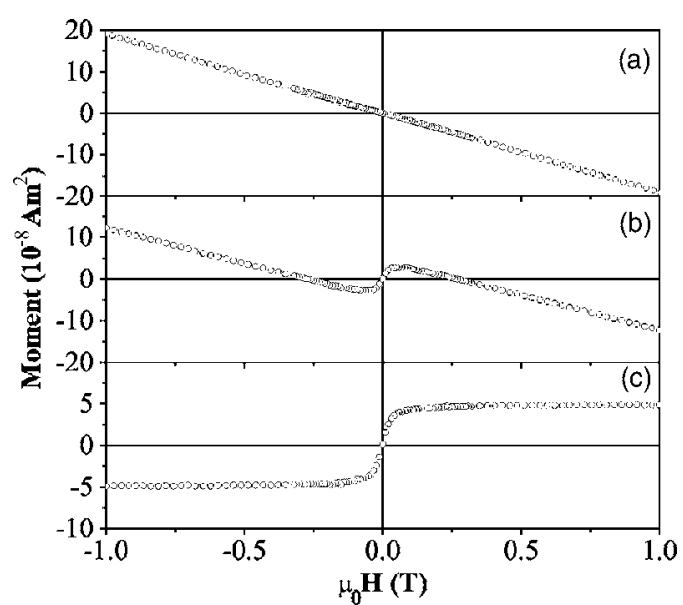

FIG. 1. Room-temperature magnetization of (a) a blank sapphire substrate, (b) $\mathrm{Zn}_{0.95} \mathrm{Co}_{0.05} \mathrm{O}$ film on sapphire, and (c) that of the $\mathrm{Zn}_{0.95} \mathrm{Co}_{0.05} \mathrm{O}$ film after subtracting the diamagnetic contribution from the substrate.

$635 \mathrm{~nm}$ during the growth process and independently calibrated by small-angle $\mathrm{x}$-ray scattering in thinner films.

The electronic properties of a homogenous film produced from a $\mathrm{Zn}_{0.95} \mathrm{Co}_{0.05} \mathrm{O}$ target and a film of $\mathrm{ZnO}$ were studied by XAS. XAS measurements were performed in both total electron yield (TEY) and total fluorescence yield (TFY) modes at beamline 7.0.1 at the Advanced Light Source (ALS), Lawrence Berkeley National Laboratory. Fluorescence yield was measured using a channeltron positioned $30^{\circ}$ directly above the incident $\mathrm{x}$-ray beam, while electron yield was recorded by the sample drain current method. In this report all the XAS spectra presented were recorded in TEY mode with no difference observed in the TFY measurements. All XAS measurements were performed in a near grazing incidence geometry with the synchrotron light incident at $\sim 20^{\circ}$ to the surface and the electric field vector of the polarized radiation at $\sim 20^{\circ}$ to the $c$ axis of the $w$ - $\mathrm{ZnO}$ which was perpendicular to the surface of the film. No contribution to the oxygen $K$ edge XAS spectrum was expected from the substrate due to the film thickness and the grazing incidence measurement geometry. This was confirmed by comparing normal versus grazing incidence spectra (TEY versus TFY), by comparison with a measured reference spectrum of the $\mathrm{Al}_{2} \mathrm{O}_{3}$ substrate, and by comparison to a thinner $(50 \mathrm{~nm})$ cobalt-doped $\mathrm{ZnO}$ film. The XAS energy scale at the oxygen $K$ edge was calibrated by comparison with reference spectra of $\mathrm{NiO}$ (Ref. 11) and by comparison with the Co metal for Co $L_{2,3}$ edges. ${ }^{12}$ The oxygen $K$ edge and cobalt $L_{2,3}$ edge XAS spectra were measured with 0.2 and $0.1 \mathrm{eV}$ energy resolutions, respectively.

Ferromagnetism at room temperature was confirmed in these cobalt-doped $\mathrm{ZnO}$ samples using a Quantum Design MPMS XL superconducting quantum interference device (SQUID) magnetometer. Shown in Fig. 1 are the roomtemperature magnetization data for a $\mathrm{Zn}_{0.95} \mathrm{Co}_{0.05} \mathrm{O}$ film measured with the field applied perpendicular to the film. ${ }^{5}$ Figure 1(a) shows the diamagnetism of a blank sapphire substrate subjected to the same thermal cycle in the deposition chamber as one with a thin film on it; the susceptibility of 4.8 $\times 10^{-9} \mathrm{~m}^{3} \mathrm{~kg}^{-1}$ agrees well with the accepted value of sap-

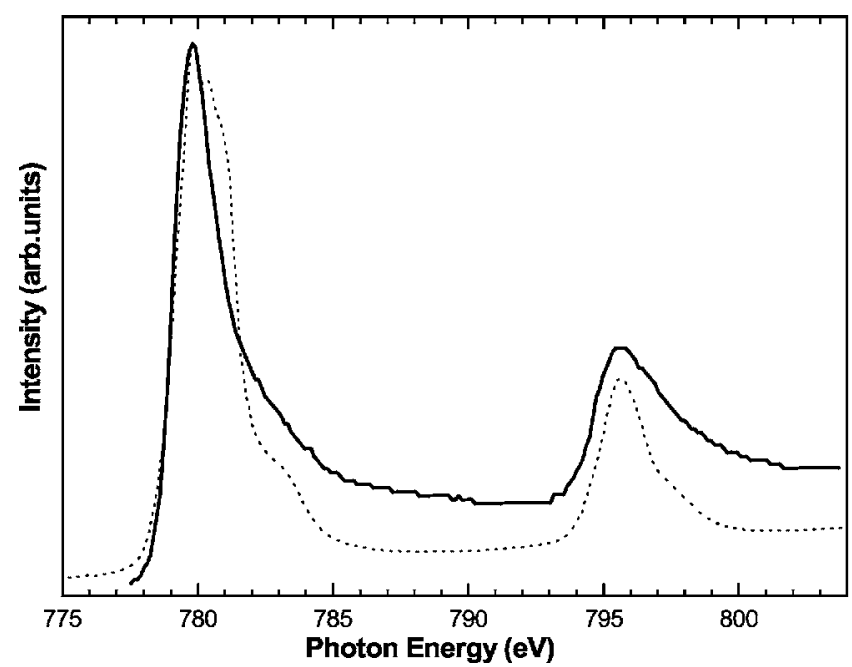

FIG. 2. Co $L_{2,3}$ edge $\mathrm{x}$-ray absorption in total electron yield of the $\mathrm{Zn}_{0.95} \mathrm{Co}_{0.05} \mathrm{O}$ (dashed line) and Co metal (solid line). The total fluorescent yield (not shown) is similar.

phire. Figure 1(b) shows the data for a Co-doped $\mathrm{ZnO}$ film on the $c$-cut sapphire substrate. The ferromagnetic signal remaining after subtraction of the linear diamagnetic background is shown in Fig. 1(c). The film is ferromagnetic with a room-temperature magnetic moment of $1.5 \mathrm{~A} \mathrm{~m}^{2} \mathrm{~kg}^{-1}$. The measured magnetization corresponds to a net ferromagnetic moment of $0.3 \mu_{B} /$ Co.

Figure 2 shows the comparison of XAS spectra of a $\mathrm{Zn}_{0.95} \mathrm{Co}_{0.05} \mathrm{O}$ film and Co metal at the Co $L_{2,3}$ edges. The $L$ edge absorption spectrum must be modeled through atomic multiplet theory to account for all allowed $2 p$ to $3 d$ transitions in the appropriate crystal field. ${ }^{13}$ The observed structure of the Co $L_{3}$ edge, in particular, is sensitive to the local symmetry and environment of the metal atom. ${ }^{13,14}$ The line shape and the spectra near the edge are similar to several of the previously reported spectra from both magnetic ${ }^{6}$ and nonmagnetic ${ }^{8}$ Co-doped $\mathrm{ZnO}$. Furthermore, the observed spectra are similar to several atomic multiplet configuration interaction calculations confirming that Co occurs in the $\mathrm{Co}^{2+}$ state in a tetrahedral crystal field. ${ }^{6,14}$ This serves to confirm that cobalt occupies the $\mathrm{Zn}$ substitutional sites in $\mathrm{ZnO}$. The spectral line shape is consistent with a theoretical spectrum calculated with a crystal-field splitting of $10 \mathrm{D} q$ $\left(T_{d}\right)=1 \mathrm{eV}$ (Ref. 14), although strictly the site symmetry in $w-\mathrm{ZnO}$ is $C_{3 v}$. The spectral line shape is completely different from that of cobalt metal; hence the possibility of extensive clustering of Co seems to be ruled out for the doped sample.

Figure 3 shows the comparison of the $\mathrm{O} K$ edge XAS spectrum from the undoped $\mathrm{ZnO}$ film and the $\mathrm{Zn}_{0.95} \mathrm{Co}_{0.05} \mathrm{O}$ film. In the undoped $\mathrm{ZnO}$ and the doped $\mathrm{ZnO}$ the spectral features are similar. The spectral features can be interpreted as follows: the region between 530 and $538 \mathrm{eV}$ can be attributed mainly to $\mathrm{O} 2 p$ hybridization with highly dispersive $\mathrm{Zn}$ $4 s$ states which form the bottom of the conduction band with the peak at $\sim 537 \mathrm{eV}$ being due to transitions to nondispersive $\mathrm{O} 2 p_{z}$ and $2 p_{x+y}$ states. The region between 539 and $550 \mathrm{eV}$ can be assigned to $\mathrm{O} 2 p$ hybridized with $\mathrm{Zn} 4 p$ states, and above $550 \mathrm{eV}$ the spectrum is due to the $\mathrm{O} 2 p$ with $\mathrm{Zn} 4 d$ hybridized states. ${ }^{15}$ However, the doped sample 


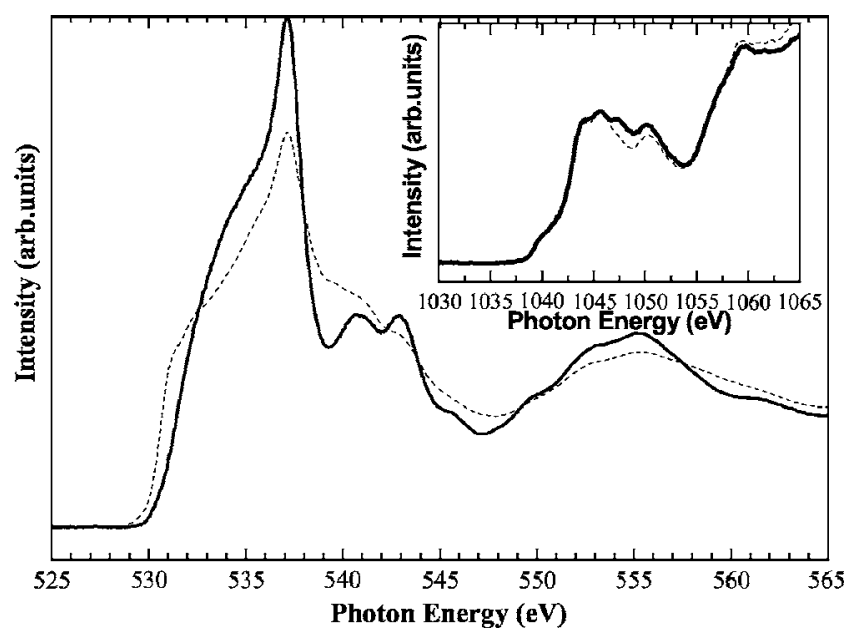

FIG. 3. O $K$ edge x-ray absorption of the undoped $\mathrm{ZnO}$ film (solid line) and $\mathrm{Zn}_{0.95} \mathrm{Co}_{0.05} \mathrm{O}$ film (dashed line). The inset shows a comparison of the $\mathrm{Zn} L_{3}$ edge XAS spectra of $\mathrm{ZnO}$ and $\mathrm{Zn}_{0.95} \mathrm{Co}_{0.05} \mathrm{O}$ films. The spectra are normalized to unit area for comparison purposes and were recorded in TEY mode.

shows two extra features at $\sim 531-532 \mathrm{eV}$, close to the conduction-band minimum where these new features can be assigned, by comparison with theoretical calculations, as being the result of cobalt $3 d$ states hybridized with $\mathrm{O} 2 p$ states. Furthermore, there is an observed difference in onset for the $\mathrm{O} K$ XAS spectrum in the two samples of $\sim 0.5 \mathrm{eV}$. This is much larger than the observed decrease in the band gap of $\sim 0.1 \mathrm{eV}$ which has been measured by optical transmission in similar Co-doped $\mathrm{ZnO}$ films grown under the same conditions. In addition, the broadening of the spectral features observed in the doped sample, particularly in relation to the peak at $\sim 537 \mathrm{eV}$, is thought to be due to the presence of oxygen vacancies. Calculations indicate that the presence of an oxygen vacancy affects the surrounding shell of the oxygen atoms by effectively altering their $1 s$ binding energies in that and subsequent shells surrounding the vacancy. In XAS the $1 s-2 p$ dipole excitation for transitions originating from these oxygen atoms (that is, in the shells surrounding a vacancy) can result in a shifting of the XAS onset to lower absorption energies. This lowering and distribution of $1 \mathrm{~s}$ binding energies also give rise to an effective broadening of all the conduction-band features as observed by XAS. It should be noted that the PLD-grown $\mathrm{ZnO}$ film has an oxygen $K$ edge XAS spectrum very similar to $\mathrm{ZnO}$ powder with no noticeable broadening of the peak at $537 \mathrm{eV}$ or a shift in the onset of the edge. ${ }^{16}$ Thus although the defect density may be expected to be similar in the two films, it could be that the presence of both the Co doping and the defects results in an exaggerated effect on the oxygen $1 s$ binding energies. (The oxygen $1 s$ binding energies in the native oxides, $\mathrm{CoO}$ and $\mathrm{ZnO}$, differ by at most $0.2 \mathrm{eV}$.)

The inset of Fig. 3 shows the $\mathrm{Zn} L_{3}$ edge absorption spectra, where there is relatively little difference between the undoped $\mathrm{ZnO}$ and the doped $\mathrm{ZnO}$. The spectral shape agrees well with the electron-energy-loss spectroscopy reports ${ }^{17}$ as well as the XAS spectrum obtained from $\mathrm{ZnO}$ nanorods ${ }^{18}$ and that of $\mathrm{ZnO}$ powder. ${ }^{16}$ Thus there appears to be no significant $\mathrm{Zn}$ defect-related features such as $\mathrm{Zn}$ interstitials. From consideration of the $\mathrm{x}$-ray-absorption measurements from both the oxygen $K$ edge and the $\mathrm{Zn} L$ edge, it might be surmised that oxygen vacancies or oxygen-related defects dominate rather than $\mathrm{Zn}$ defects such as interstitials.

It is known that certain atomic defects in ionic crystals, such as oxygen vacancies with trapped electrons or holes, may have low-lying triplet states that overlap to form an impurity band. ${ }^{19}$ It is suggested that the magnetic defects may interact by long-range interactions mediated by the impurity band to give a ferromagnetic ground state. ${ }^{10}$ These XAS observations provide further evidence that the observed magnetic moment is consistent with the spin split impurity band model predicted by Coey et al. ${ }^{10}$

These spectroscopic measurements on undoped $\mathrm{ZnO}$ and Co-doped $\mathrm{ZnO}$ PLD-grown films exhibit material-specific defect features in the oxygen spectra. Also, this is an experimental evidence from the oxygen $K$ edge x-ray absorption that shows the combined effects of the hybridized Co states and oxygen vacancies. The shift in the onset of the oxygen $K$ edge XAS of $0.5 \mathrm{eV}$ is proposed to be due to the presence of oxygen vacancies, a view reinforced when considered in conjunction with optical measurements that exhibit a bandgap narrowing of only $0.1 \mathrm{eV}$. The cobalt substituted in the zinc cation site and the presence of oxygen vacancies may be jointly responsible for the observed ferromagnetism. Much work needs to be done to identify the nature of these defects and to understand better the magnetism of these magnetic materials.

The authors wish to acknowledge financial support of the Trinity College IAMS PRTLI program, of Science Foundation Ireland, and also of Enterprise Ireland through the International Collaboration Travel Fund. The Advanced Light Source is supported by the Director, Office of Science, Office of Basic Energy Sciences, of the U.S. Department of Energy under Contract No. DE-AC02-05CH11231. The Boston University program is supported in part by the U.S. Department of Energy under Contract No. DE-FG0298ER45680. One of the authors (L.S.D.) is supported by the Irish Research Council for Science, Engineering and Technology. Another author (T.L.) acknowledges support from the ALS Doctoral Fellowship Program. Another author (T.S.) acknowledges financing through postdoctoral fellowship from Göran Gustafsson Foundation.

\footnotetext{
${ }^{1}$ T. Dietl et al.,Science 287, 1019 (2000).

${ }^{2}$ K. Ueda, H. Tabata, and T. Kawai, Appl. Phys. Lett. 79, 988 (2001).

${ }^{3}$ S. Ramachandran et al., J. Electron. Mater. 33, 1298 (2004).

${ }^{4}$ K. Rode et al., J. Appl. Phys. 93, 7676 (2003).

${ }^{5}$ M. Venkatesan et al., Phys. Rev. Lett. 93, 177206 (2004).

${ }^{6}$ J. Okabayashi et al., J. Appl. Phys. 95, 3573 (2004).

${ }^{7}$ J. H. Kim et al.,J. Appl. Phys. 92, 6066 (2002).

${ }^{8}$ S. C. Wi et al., Appl. Phys. Lett. 84, 4233 (2004).

${ }^{9}$ K. Sato et al., Jpn. J. Appl. Phys., Part 2 40, L334 (2001).

${ }^{10}$ J. M. D. Coey et al.,Nat. Mater. 4, 173 (2005).

${ }^{11}$ F. M. F. de Groot et al., Phys. Rev. B 40, 5715 (1989).

${ }^{12}$ C. Mitterbauer et al., Ultramicroscopy 96, 469 (2003).

${ }^{13}$ F. M. F. de Groot et al., J. Phys.: Condens. Matter 5, 2277 (1993)

${ }^{14}$ G. van der Laan et al., J. Phys.: Condens. Matter 4, 4189 (1992).

${ }^{15}$ J. H. Guo et al., J. Phys.: Condens. Matter 14, 6969 (2002).

${ }^{16}$ C. McGuinness et al.,Phys. Rev. B 68, 165104 (2003).

${ }^{17}$ T. Mizoguchi et al.,Phys. Rev. B 70, 045103 (2004).

${ }^{18}$ J. W. Chiou et al., Appl. Phys. Lett. 85, 3220 (2004).

${ }^{19}$ A. M. Stoneham, Theory of Defects in Solids (Clarendon, Oxford, 1975).
} 